\title{
Low energy kaon-hyperon interaction
}

\author{
M. G. L. N. Santos ${ }^{*}$ and C. C. Barros, Jr. ${ }^{\dagger}$ \\ Departamento de Física, CFM, Universidade Federal de Santa Catarina, Florianópolis SC, CEP 88010-900, Brazil
}

(Received 6 October 2018; published 26 February 2019)

\begin{abstract}
In this work we study the low energy kaon-hyperon interaction, considering effective chiral Lagrangians that include kaons, $\sigma$ mesons, hyperons, and the corresponding resonances. We calculate the scattering amplitudes, and then the total cross sections, angular distributions, polarizations, and the $S$ and $P$ phase shifts.
\end{abstract}

DOI: 10.1103/PhysRevC.99.025206

\section{INTRODUCTION}

A subject that is very interesting and remains not very well studied is low energy hyperon interactions. Despite the fact that experimental data for many hyperon processes are not available (for example the $K \Lambda$ and $K \Omega$ interactions) and that on the theoretical side they are not fully described, this kind of interaction is a fundamental element for several physical systems of interest.

In the study of hypernuclei structure [1-4], the knowledge of nucleon-hyperon and hyperon-hyperon interactions is an essential aspect. In order to understand these interactions, and to determine the potentials of interest, an accurate understanding of meson-hyperon interactions is needed.

Another system where hyperon interactions are required is in the study of hyperon stars. Since the proposal of the hypothesis that hyperons could be produced inside neutron stars at high densities, many models have been proposed, as for example in [5-8], and the effect of the presence of hyperons in the equations of state, and consequently in the determination of the star masses, has been studied. The indeterminations in the nucleon-hyperon and hyperon-hyperon potentials cause difficulties in the understanding of these stars.

In high energy physics also this kind of interaction is very important. When studying hyperon polarization, produced in proton-nucleus and nucleus-nucleus collisions [9-18], in [19-21] the final interactions of the hyperons and antihyperons with the produced pions are a central ingredient in order to explain the final polarizations. It has been shown that the effect of the hyperon interactions with the surrounding hot medium, composed predominantly of pions, is very important. The observed differences between the polarizations of hyperons and antihyperons are very difficult to explain in another

\footnotetext{
*magwwo@gmail.com

†barros.celso@ufsc.br
}

Published by the American Physical Society under the terms of the Creative Commons Attribution 4.0 International license. Further distribution of this work must maintain attribution to the author(s) and the published article's title, journal citation, and DOI. Funded by $S C O A P^{3}$. way. The effect of the final kaon-hyperon interactions has not been considered yet, and it may cause corrections in the final polarization. For this reason, this work is very important and this effect must be investigated. Recent results from BNL Relativistic Heavy Ion Collider (RHIC) [22] and even the hyperons produced in the CERN Large Hadron Collider (LHC) may be studied in a similar form, and in order to obtain accurate results these interactions must be considered.

For these reasons, this work will be devoted to the study of the kaon-hyperon $(K Y)$ and antikaon-hyperon $(\bar{K} Y)$ interactions. This work may be considered as a continuation of the study proposed in [23-25], where the pion-hyperon interactions have been described with a model based on effective chiral Lagrangians where the exchange of mesons and baryons has been taken into account. In this model [23], the resonances dominate many channels of the reactions, as may be seen in the results. This behavior may be considered as an experimental feature, similar to what happens in the low energy pion-nucleon interactions, where the isospin-3/2 and spin-3/2 channels are dominated by the $\Delta^{++}$resonance. Comparison with the data from the HyperCP experiment $[26,27]$ shows a very good accord with the results obtained for the $\pi \Lambda$ scattering in [23]. So, the work that will be shown in this paper is based on the ideas presented in this model.

This paper will present the following content: in Sec. II, the basic formalism will be shown. in Secs. III, IV, V, and VI, we study the kaon-lambda $(K \Lambda)$, antikaon-lambda $(\bar{K} \Lambda)$, kaon-sigma $(K \Sigma)$, and antikaon-sigma $(\bar{K} \Sigma)$ interactions. We present discussions and conclusions in Sec. VII. In the Appendix, some expressions of interest will be presented.

\section{THE METHOD}

In order to study $K Y$ and $\bar{K} Y$ interactions, we will use a model proposed with the purpose of studying low energy pion-hyperon interactions [23-25] that is based on an analogy with models successfully used to describe $\pi N$ interactions considering chiral effective Lagrangians. These interactions are very well studied, as for example in [28-31], both theoretically, where many models have been proposed, and experimentally, with a large amount of experimental data available. A basic characteristic of this system is the dominance of resonances in the scattering amplitudes at low energies. The 
$\Delta^{++}$, for example, dominates the cross section of the $\pi^{+} p$ scattering at low energies. As this particle has spin $3 / 2$ and isospin $3 / 2$, it may be introduced in the theory by considering a Lagrangian in the form of Eq. (2). In the study of pion-hyperon interactions [23], a similar behavior has been observed, so we expect that in $K Y$ interactions it also occurs.

In this section we will present the basic formalism that will be used to study kaon-hyperon interactions (it is the same one worked out in the study pion-hyperon interactions [23]) and how the observables may be obtained. In the method that will be followed in this work, some important characteristics of the interacting particles will be implemented: the spin, the isospin, and the mass of each particle. These characteristics determine which Lagrangians have to be used in order to build the model.

For example, in [28], the Lagrangians considered to study the $\pi N$ scattering are given by

$$
\begin{aligned}
\mathcal{L}_{\pi N N} & =\frac{g}{2 m}\left(\bar{N} \gamma_{\mu} \gamma_{5} \vec{\tau} N\right) \cdot \partial^{\mu} \vec{\phi}, \\
\mathcal{L}_{\pi N \Delta} & =g_{\Delta}\left\{\bar{\Delta}^{\mu}\left[g_{\mu \nu}-\left(Z+\frac{1}{2}\right) \gamma_{\mu} \gamma_{\nu}\right] \vec{M} N\right\} \cdot \partial^{\nu} \vec{\phi},
\end{aligned}
$$

where $N, \Delta$, and $\vec{\phi}$ are the nucleon, delta, and pion fields with masses $m, m_{\Delta}$, and $m_{\pi}$, respectively, $\vec{M}$ and $\vec{\tau}$ are the isospin recombination matrices, and $Z$ is a parameter representing the possibility of the off-shell $\Delta$ having spin $1 / 2$. The parameters $g$ and $g_{\Delta}$ are the coupling constants. In [23] similar Lagrangians have been used to study the pion-hyperon interactions, and in this work the same procedure will be adopted. So, in the following sections these Lagrangians will be adapted to the kaon-hyperon systems.

Calculating the diagrams, considering the interactions described by the Lagrangians above for an arbitrary process, the scattering amplitudes may be written in the form

$$
T_{\pi N}^{\beta \alpha}=\sum_{I} T^{I}\left\langle\beta\left|P_{I}\right| \alpha\right\rangle=\sum_{I} T^{I} P_{I}^{\beta \alpha},
$$

that is, a sum over all the $I$ isospin states where $P_{I}$ is a projection operator; the indices $\alpha$ and $\beta$ are relative to the initial and final isospin states of the $\pi$, and $T^{I}$ is an amplitude for a given isospin state that may be written as

$$
T^{I}=\bar{u}\left(\overrightarrow{p^{\prime}}\right)\left[A^{I}+\frac{1}{2}\left(\not k+\not k^{\prime}\right) B^{I}\right] u(\vec{p}),
$$

where $u(\vec{p})$ is a spinor representing the initial baryon, incoming with four-momentum $p_{\mu}$. The final baryon has a spinor $\bar{u}\left(\overrightarrow{p^{\prime}}\right)$, four-momentum $p_{\mu}^{\prime}$, and $k_{\mu}$ and $k_{\mu}^{\prime}$ are the incoming and outgoing meson four-momenta. The amplitudes $A^{I}$ and $B^{I}$ are calculated from the diagrams. So, if these amplitudes are determined, the $T^{I}$ amplitudes may be obtained and then we will be able to compute the observables of interest.

The scattering matrix for an isospin state is given by the expression

$$
M^{I}=\frac{T^{I}}{8 \pi \sqrt{s}},
$$

which may be decomposed into the spin-non-flip and spinflip amplitudes $f^{I}(k, \theta)$ and $g^{I}(k, \theta)$, defined in terms of the momentum $k=|\vec{k}|$ and $x=\cos \theta$, with $\theta$ the scattering angle, as

$$
M^{I}=f^{I}(k, x)+g^{I}(k, x) \vec{\sigma} \cdot \hat{n},
$$

where $\hat{n}$ is a vector normal to the scattering plane, and may be expanded in terms of the partial-wave amplitudes $a_{l \pm}$ with

$$
\begin{aligned}
& f^{I}(k, x)=\sum_{l=0}^{\infty}\left[(l+1) a_{l+}^{I}(k)+l a_{l-}^{I}(k)\right] P_{l}(x), \\
& g^{I}(k, x)=i \sum_{l=1}^{\infty}\left[a_{l-}^{I}(k)-a_{l+}^{I}(k)\right] P_{l}^{(1)}(x) .
\end{aligned}
$$

These amplitudes may be calculated using the Legendre polynomials orthogonality relations

$$
a_{l \pm}^{I}(k)=\frac{1}{2} \int_{-1}^{1}\left[P_{l}(x) f_{1}^{I}(k, x)+P_{l \pm 1}(x) f_{2}^{I}(k, x)\right] d x,
$$

with

$$
\begin{aligned}
& f_{1}^{I}(k, x)=\frac{(E+m)}{8 \pi \sqrt{s}}\left[A^{I}+(\sqrt{s}-m) B^{I}\right], \\
& f_{2}^{I}(k, x)=\frac{(E-m)}{8 \pi \sqrt{s}}\left[-A^{I}+(\sqrt{s}+m) B^{I}\right],
\end{aligned}
$$

where $E$ is the baryon energy in the center-of-mass frame and $\sqrt{s}$ is given by a Mandelstam variable (see the Appendix). At low energies the $S(l=0)$ and $P(l=1)$ waves dominate the scattering amplitudes, and for higher values of $l$ the amplitudes are much smaller (almost negligible), so they may be considered as small corrections.

Calculating the amplitudes at the tree level, the results obtained will be real, and then violate the unitarity of the $S$ matrix. As is usually done, we may reinterpret these results as elements of the $K$ reaction matrix [23-25] and then obtain unitarized amplitudes

$$
a_{l \pm}^{U}=\frac{a_{l \pm}}{1-i k a_{l \pm}} .
$$

The differential cross sections may be calculated using the previous results,

$$
\frac{d \sigma}{d \Omega}=|f|^{2}+|g|^{2},
$$

and integrating this expression over the solid angle we obtain the total cross sections

$$
\sigma_{T}=4 \pi \sum_{l}\left[(l+1)\left|a_{l+}^{U}\right|^{2}+l\left|a_{l-}^{U}\right|^{2}\right],
$$

and the phase shifts are given by

$$
\delta_{l \pm}=\tan ^{-1}\left(k a_{ \pm}\right) .
$$

An important task to achieve is to determine the coupling constant for each resonance that will be considered. We will adopt the same procedure considered in [23], comparing the amplitude obtained in the calculations with the relativistic Breit-Wigner expression, that is determined in terms of ex- 
(a)

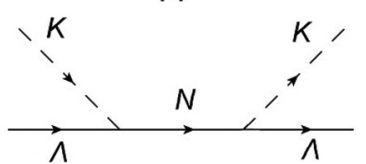

(c)
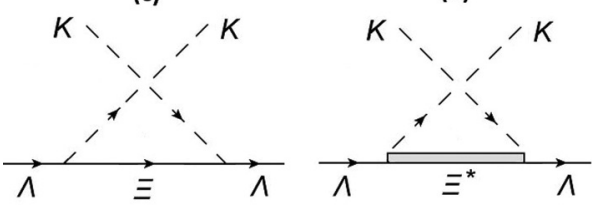

(d)

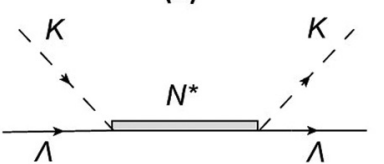

(e)

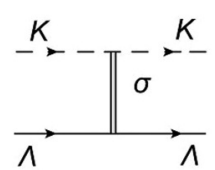

FIG. 1. Diagrams for the $K \Lambda$ interaction.

perimental quantities

$$
\delta_{l \pm}=\tan ^{-1}\left[\frac{\Gamma_{0}\left(\frac{k}{k_{0}}\right)^{2 J+1}}{2\left(m_{r}-\sqrt{s}\right)}\right],
$$

where $\Gamma_{0}$ is the width, $k_{0}=\left|\overrightarrow{k_{0}}\right|$ is the momentum at the peak of the resonance in the center-of-mass system, $m_{r}$ is its mass, and $J$ the angular momentum, considering the data from [32]. We will consider the coupling constant that better fits this expression in each case.

In the following sections we will apply this formalism in the study of the reactions of interest.

\section{KAON-LAMBDA INTERACTION}

Since the $\Lambda$ hyperon has isospin 0 , the scattering amplitude for the $K \Lambda$ interaction will have the form

$$
T_{K \Lambda}=\bar{u}\left(\overrightarrow{p^{\prime}}\right)\left[A(k, \theta)+\left(\frac{\not k+\not k^{\prime}}{2}\right) B(k, \theta)\right] u(\vec{p}),
$$

with the variables defined in Sec. II. Comparing this expression with (3), we have a simple result, $P_{1 / 2}^{\beta \alpha}=1$, as the kaon has isospin $1 / 2$, and just one isospin amplitude.

In Fig. 1 we show the diagrams and the particles considered to formulate the $K \Lambda$ interaction. The particles considered for each diagram are shown in Table I.

For the calculation of the contribution of particles with spin-1/2 $(N$ and $\Xi)$ in the intermediate state [Figs. 1(a) and 1(c)], the Lagrangian of interaction is [considering the necessary adaptations from Eq. (1)]

$$
\mathcal{L}_{\Lambda K B}=\frac{g_{\Lambda K B}}{2 m_{\Lambda}}\left(\bar{B} \gamma_{\mu} \gamma_{5} \Lambda\right) \partial^{\mu} \phi+\text { H.c. }
$$

TABLE I. Particles considered in the $K \Lambda$ interaction.

\begin{tabular}{lccc}
\hline \hline & $J^{\pi}$ & $I$ & Mass $(\mathrm{MeV})$ \\
\hline$N$ & $1 / 2^{+}$ & $1 / 2$ & 938 \\
$N(1650)$ & $1 / 2^{-}$ & $1 / 2$ & 1650 \\
$N(1710)$ & $1 / 2^{+}$ & $1 / 2$ & 1710 \\
$N^{*}(1875)$ & $3 / 2^{-}$ & $1 / 2$ & 1875 \\
$N^{*}(1900)$ & $3 / 2^{+}$ & $1 / 2$ & 1900 \\
$\Xi$ & $1 / 2^{+}$ & $1 / 2$ & 1320 \\
$\Xi^{*}(1820)$ & $3 / 2^{-}$ & $1 / 2$ & 1820 \\
\hline \hline
\end{tabular}

where $\phi$ represents the kaon field, $B$ the intermediate baryon field with mass $m_{B}$, and $\Lambda$ the hyperon field, with mass $m_{\Lambda}$.

Calculating the Feynman diagrams and comparing with Eq. (17), we find the amplitudes for the $N$ (spin-1/2) particles contribution

$$
\begin{aligned}
A_{N} & =\frac{g_{\Lambda K N}^{2}}{4 m_{\Lambda}^{2}}\left(m_{N}+m_{\Lambda}\right)\left(\frac{s-m_{\Lambda}^{2}}{s-m_{N}^{2}}\right), \\
B_{N} & =-\frac{g_{\Lambda K N}^{2}}{4 m_{\Lambda}^{2}}\left[\frac{2 m_{\Lambda}\left(m_{\Lambda}+m_{N}\right)+s-m_{\Lambda}^{2}}{s-m_{N}^{2}}\right],
\end{aligned}
$$

and for the $\Xi$ (spin-1/2) hyperon in the crossed diagram [Fig. 1(c)] the contribution is

$$
\begin{aligned}
& A_{\Xi}=\frac{g_{\Lambda K \Xi}^{2}}{4 m_{\Lambda}^{2}}\left(m_{\Xi}+m_{\Lambda}\right)\left(\frac{u-m_{\Lambda}^{2}}{u-m_{\Xi}^{2}}\right), \\
& B_{\Xi}=\frac{g_{\Lambda K \Xi}^{2}}{4 m_{\Lambda}^{2}}\left[\frac{2 m_{\Lambda}\left(m_{\Lambda}+m_{\Xi}\right)+u-m_{\Lambda}^{2}}{u-m_{\Xi}^{2}}\right],
\end{aligned}
$$

where $s$ and $u$ are Mandelstam variables, defined in the Appendix, and $g_{\Lambda K N(\Xi)}$ are the coupling constants.

In a similar way, we adapted the interaction Lagrangian (2) for the exchange of spin-3/2 resonances, shown in Figs. 1(b) and $1(\mathrm{~d})$ :

$$
\mathcal{L}_{\Lambda K B^{*}}=g_{\Lambda K B^{*}}\left\{\bar{B}^{* \mu}\left[g_{\mu \nu}-\left(Z+\frac{1}{2}\right) \gamma_{\mu} \gamma_{\nu}\right] \Lambda\right\} \partial^{v} \phi+\text { H.c. }
$$

Calculating the amplitude for the exchange of a spin- $3 / 2$ $N^{*}$ [Fig. 1(b)] we have

$$
\begin{aligned}
& A_{N^{*}}=\frac{g_{\Lambda K N^{*}}^{2}}{6}\left[\frac{2 \hat{A}+3\left(m_{\Lambda}+m_{N^{*}}\right) t}{m_{N^{*}}^{2}-s}+a_{0}\right], \\
& B_{N^{*}}=\frac{g_{\Lambda K N^{*}}^{2}}{6}\left[\frac{2 \hat{B}+3 t}{m_{N^{*}}^{2}-s}-b_{0}\right]
\end{aligned}
$$

where

$$
\begin{aligned}
\hat{A}= & 3\left(m_{\Lambda}+m_{N^{*}}\right)\left(q_{N^{*}}\right)^{2}+\left(m_{N^{*}}-m_{\Lambda}\right)\left(E_{N^{*}}+m_{\Lambda}\right)^{2}, \\
\hat{B}= & 3\left(q_{N^{*}}\right)^{2}-\left(E_{N^{*}}+m_{\Lambda}\right)^{2}, \\
a_{0}= & -\frac{\left(m_{\Lambda}+m_{N^{*}}\right)}{m_{N^{*}}^{2}}\left(2 m_{N^{*}}^{2}+m_{\Lambda} m_{N^{*}}-m_{\Lambda}^{2}+2 m_{K}^{2}\right) \\
& +\frac{4}{m_{N^{*}}^{2}}\left[\left(m_{N^{*}}+m_{\Lambda}\right) Z+\left(2 m_{N^{*}}+m_{\Lambda}\right) Z^{2}\right]\left[s-m_{\Lambda}^{2}\right], \\
b_{0}= & \frac{8}{m_{N^{*}}^{2}}\left[\left(m_{\Lambda}^{2}+m_{\Lambda} m_{N^{*}}-m_{K}^{2}\right) Z+\left(2 m_{\Lambda} m_{N^{*}}+m_{\Lambda}^{2}\right) Z^{2}\right] \\
& +\frac{\left(m_{\Lambda}+m_{N^{*}}\right)^{2}}{m_{N^{*}}^{2}}+\frac{4 Z^{2}}{m_{N^{*}}^{2}}\left[s-m_{\Lambda}^{2}\right] .
\end{aligned}
$$


TABLE II. Parameters for the $K \Lambda$ interaction.

\begin{tabular}{ll}
\hline \hline$m_{\pi}$ & $140 \mathrm{MeV}$ \\
$m_{K}$ & $496 \mathrm{MeV}$ \\
$m_{\Lambda}$ & $1116 \mathrm{MeV}$ \\
$Z$ & -0.5 \\
$g_{\Lambda K N}$ & 11.50 \\
$g_{\Lambda K N(1650)}$ & $9.90 \mathrm{GeV}^{-1}$ \\
$g_{\Lambda K N(1710)}$ & $5.20 \mathrm{GeV}^{-1}$ \\
$g_{\Lambda K N^{*}(1875)}$ & $0.53 \mathrm{GeV}^{-1}$ \\
$g_{\Lambda K N^{*}(1900)}$ & $2.60 \mathrm{GeV}^{-1}$ \\
$g_{\Lambda K \Xi}$ & 0.24 \\
$g_{\Lambda K \Xi^{*}(1820)}$ & $1.80 \mathrm{GeV}^{-1}$ \\
\hline \hline
\end{tabular}

For the spin-3/2 $\Xi^{*}$ resonance [Fig. 1(d)], the amplitudes are

$$
\begin{aligned}
& A_{\Xi^{*}}=\frac{g_{\Lambda K \Xi^{*}}^{2}}{6}\left[\frac{2 \hat{A}^{\prime}+3\left(m_{\Lambda}+m_{\Xi^{*}}\right) t}{m_{\Xi^{*}}^{2}-u}+c_{0}+c_{z}\left(u-m_{\Lambda}^{2}\right)\right], \\
& B_{\Xi^{*}}=\frac{g_{\Lambda K \Xi^{*}}^{2}}{6}\left[-\frac{2 \hat{B}^{\prime}+3 t}{m_{\Xi^{*}}^{2}-u}+d_{0}+d_{z}\left(u-m_{\Lambda}^{2}\right)\right],
\end{aligned}
$$

where

$$
\begin{aligned}
c_{0}= & -\frac{\left(m_{\Lambda}+m_{\Xi^{*}}\right)}{m_{\Xi^{*}}^{2}}\left(2 m_{\Xi^{*}}^{2}+m_{\Lambda} m_{\Xi^{*}}-m_{\Lambda}^{2}+2 m_{K}^{2}\right) \\
c_{z}= & \frac{4}{m_{\Xi^{*}}^{2}}\left[\left(m_{\Xi^{*}}+m_{\Lambda}\right) Z+\left(2 m_{\Xi^{*}}+m_{\Lambda}\right) Z^{2}\right] \\
d_{0}= & \frac{8}{m_{\Xi^{*}}^{2}}\left[\left(m_{\Lambda}^{2}+m_{\Lambda} m_{\Xi^{*}}-m_{K}^{2}\right) Z+\left(2 m_{\Lambda} m_{\Xi^{*}}+m_{\Lambda}^{2}\right) Z^{2}\right] \\
& +\frac{\left(m_{\Lambda}+m_{\Xi^{*}}\right)^{2}}{m_{\Xi^{*}}^{2}} \\
d_{z}= & \frac{4 Z^{2}}{m_{\Xi^{*}}^{2}}
\end{aligned}
$$

where $t, q_{N^{*}}, E_{N^{*}}$ are defined in the Appendix and $m_{K}, m_{N^{*}}$ * are the kaon and the $N^{*}$ masses respectively. For $\hat{A}^{\prime}$ and $\hat{B}^{\prime}$ we change the $N^{*}$ parameters, inserting the $\Xi^{*}$ ones in Eqs. (26) and (27). $g_{\Lambda K N^{*}\left(\Xi^{*}\right)}$ are the coupling constants.

For the last diagram, Fig. 1(e), the scalar $\sigma$ meson exchange, a parametrization of the amplitude has been considered [23-25]:

$$
\begin{aligned}
A_{\sigma} & =a+b t, \\
B_{\sigma} & =0,
\end{aligned}
$$

with $a=1.05 m_{\pi}^{-1}, b=-0.8 m_{\pi}^{-3}$ and the pion mass $m_{\pi}$. Some discussions about this term may be found in [24,33-36].

The parameters considered in the $K \Lambda$ interaction are shown in Table II; the masses are taken from [32].

The coupling constants $g_{\Lambda K N}$ and $g_{\Lambda K \Xi}$ are determined in (38) and (39), but the other coupling constants of the model must be determined. Considering SU(3) in the quark model,

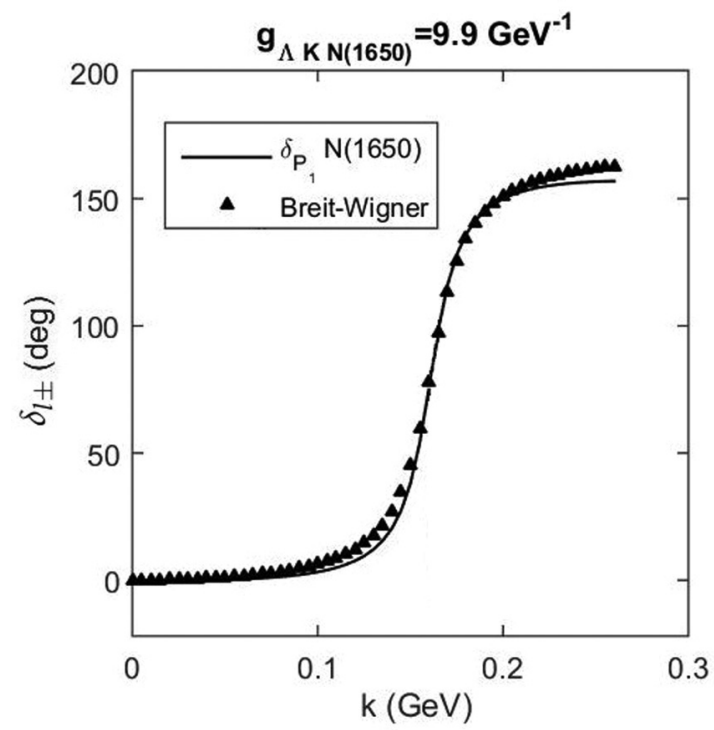

FIG. 2. Breit-Wigner fit for the $N(1650)$ resonance.

we have the relations $[37,38]$

$$
\begin{aligned}
& g_{\Lambda K N}=-\frac{1}{\sqrt{3}} f(1+2 \alpha), \\
& g_{\Lambda K \Xi}=-\frac{1}{\sqrt{3}} f(4 \alpha-1), \\
& g_{\Sigma K N}=f(1-2 \alpha), \\
& g_{\Sigma K \Xi}=f,
\end{aligned}
$$

where $f=g_{\pi N N}=13.40$ [39], and from the relation $g_{\pi \Lambda \Sigma}=$ $2 f(1-\alpha) / \sqrt{3}$, with $g_{\pi \Lambda \Sigma}=11.70$ [40], we find $\alpha=0.244$, which is in accord with the value found in [38].

For higher mass particle exchanges we consider the fact that the scattering amplitudes are dominated by these resonances when the energies are close to their masses, and then the phase shifts have the approximate form of the Breit-Wigner expression (16), so, by comparing the calculated amplitudes with Eq. (16), the coupling constants may be determined in the same way it was done in [23]. Figure 2 shows one example. The obtained results are shown in Table II.

In Figure 3, we show our results for the total elastic cross section and the phase shifts as functions of the kaon momentum $k$, defined in the center-of-mass frame.

Observing the figure, we can note that the resonances, and especially the $N(1650)$ contribution, dominate the total cross section when $k \sim 150 \mathrm{MeV}$, as expected. At higher energies, the other resonances also have an important effect.

\section{ANTIKAON-LAMBDA INTERACTION}

The $\bar{K} \Lambda$ interations may be studied exactly in the same way as was done in the last section for the the $K \Lambda$ interactions. Now we have the contributions presented in Fig. 4, where the Lagrangians take into account the $N, \Xi, \Lambda$, and $\phi^{\prime}$ (represent- 

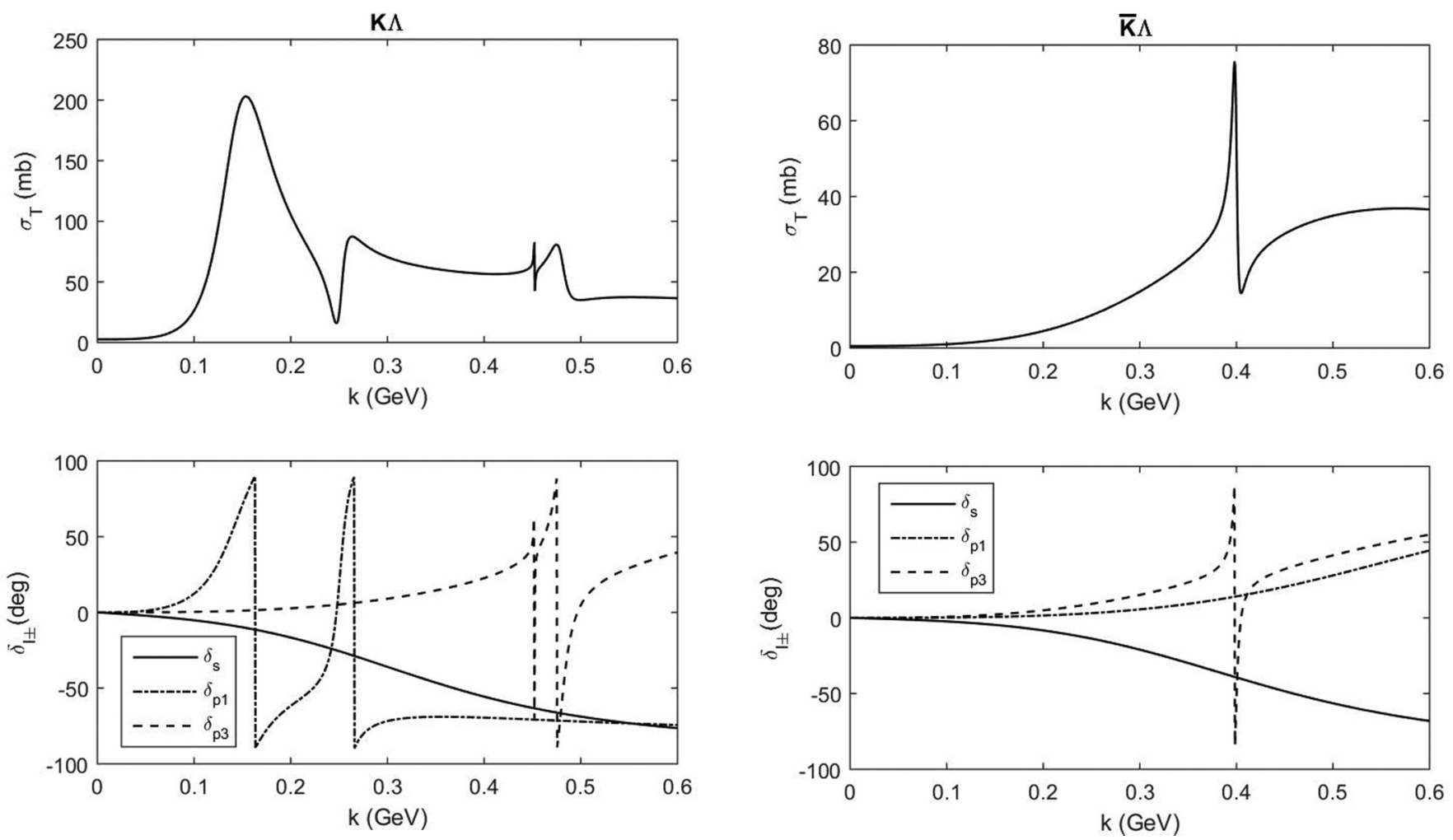

FIG. 3. Total cross section and phase shifts of the $K \Lambda$ scattering.

ing the antikaon) fields:

$$
\begin{aligned}
\mathcal{L}_{\Lambda \bar{K} B} & =\frac{g_{\Lambda \bar{K} B}}{2 m_{\Lambda}}\left(\bar{B} \gamma_{\mu} \gamma_{5} \Lambda\right) \partial^{\mu} \phi^{\prime}, \\
\mathcal{L}_{\Lambda \bar{K} B^{*}} & =g_{\Lambda \bar{K} B^{*}}\left\{\bar{B}^{* \mu}\left[g_{\mu \nu}-\left(Z+\frac{1}{2}\right) \gamma_{\mu} \gamma_{\nu}\right] \Lambda\right\} \partial^{\nu} \phi^{\prime} .
\end{aligned}
$$

The parameters considered are those given before, $m_{\bar{K}}=$ $m_{K}$, and for the crossed diagrams in Figs. 4(c) and 4(d) we have considered only $N(938), N(1650)$, and $N^{*}(1900)$, which are the most important processes. The amplitudes (36) and (37) have been calculated and the results are shown in Fig. 5.

(a)

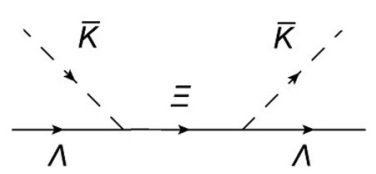

(c)
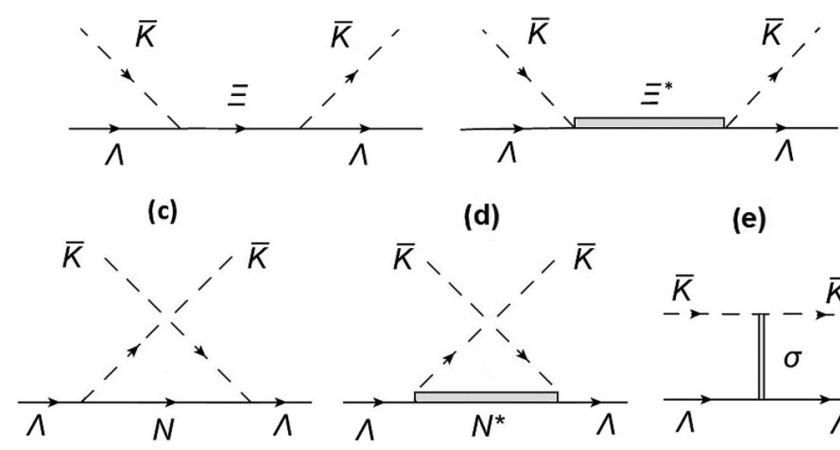

(d)

(e)

FIG. 4. Diagrams for the $\bar{K} \Lambda$ interaction.

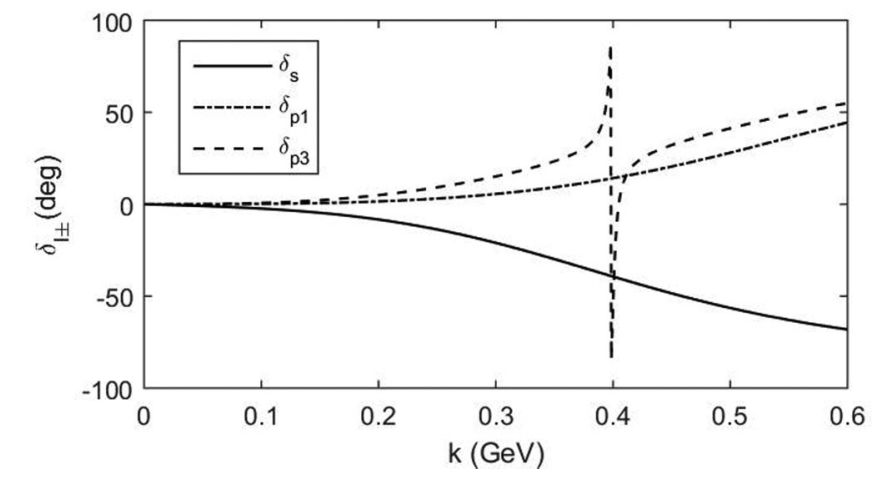

FIG. 5. Total cross section and phase shifts of the $\bar{K} \Lambda$ scattering.

\section{KAON-SIGMA INTERACTION}

In this case the interacting particles have isospins $1 / 2$ and 1 ( $K$ and $\Sigma$ respectively). So, we have two possible total isospin states, $1 / 2$ and $3 / 2$, which allow also the exchange of $\Delta$ particles.

The scattering amplitude has the general form

$$
\begin{aligned}
T_{K \Sigma}^{\beta \alpha}= & \bar{u}\left(\overrightarrow{p^{\prime}}\right)\left\{\left[A^{+}+\left(\frac{\not k+\not k^{\prime}}{2}\right) B^{+}\right] \delta^{\beta \alpha}\right. \\
& \left.+\left[A^{-}+\left(\frac{\not k+\not k^{\prime}}{2}\right) B^{-}\right] i \epsilon^{\beta \alpha c} \tau_{c}\right\} u(\vec{p}),
\end{aligned}
$$

and the considered projection operators are

$$
\begin{aligned}
& P_{\frac{1}{2}}^{\beta \alpha}=\frac{1}{3} \delta^{\beta \alpha}+\frac{i}{3} \epsilon^{\beta \alpha c} \tau_{c}, \\
& P_{\frac{3}{2}}^{\beta \alpha}=\frac{2}{3} \delta^{\beta \alpha}-\frac{i}{3} \epsilon^{\beta \alpha c} \tau_{c},
\end{aligned}
$$

where the indices $\alpha$ and $\beta$ are relative to the initial and final isospin states of the $\Sigma$.

The contributing diagrams are shown in Fig. 6 and the considered particles in Table III. The Lagrangians (1) and (2) now become

$$
\begin{aligned}
\mathcal{L}_{\Sigma K B} & =\frac{g_{\Sigma K B}}{2 m_{\Sigma}}\left(\bar{B} \gamma_{\mu} \gamma_{5} \vec{\tau} \cdot \vec{\Sigma}\right) \partial^{\mu} \phi, \\
\mathcal{L}_{\Sigma K B^{*}} & =g_{\Sigma K B^{*}}\left\{\bar{B}^{* \mu}\left[g_{\mu \nu}-\left(Z+\frac{1}{2}\right) \gamma_{\mu} \gamma_{\nu}\right] \vec{Q} \cdot \vec{\Sigma}\right\} \partial^{\nu} \phi,
\end{aligned}
$$



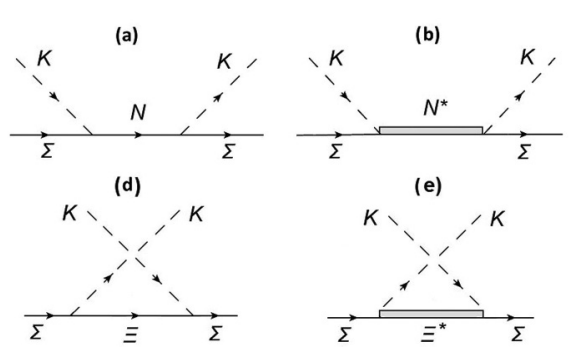

(e)

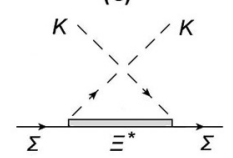

FIG. 6. Diagrams for the $K \Sigma$ interaction.

where $\vec{Q}$ is the $\vec{M}$ matrix for $\Delta(I=3 / 2)$ or $\vec{\tau}$ matrix for the $N^{*}$ and $\Xi^{*}(I=1 / 2)$.

The resulting amplitudes for nucleons in the intermediate state [Fig. 6(a)] are

$$
\begin{aligned}
& A_{N}^{+}=\frac{g_{\Sigma K N}^{2}}{4 m_{\Sigma}^{2}}\left(m_{N}+m_{\Sigma}\right)\left(\frac{s-m_{\Sigma}^{2}}{s-m_{N}^{2}}\right), \\
& B_{N}^{+}=-\frac{g_{\Sigma K N}^{2}}{4 m_{\Sigma}^{2}}\left[\frac{2 m_{\Sigma}\left(m_{\Sigma}+m_{N}\right)+s-m_{\Sigma}^{2}}{s-m_{N}^{2}}\right], \\
& A_{N}^{-}=\frac{g_{\Sigma K N}^{2}}{4 m_{\Sigma}^{2}}\left(m_{N}+m_{\Sigma}\right)\left(\frac{s-m_{\Sigma}^{2}}{s-m_{N}^{2}}\right), \\
& B_{N}^{-}=-\frac{g_{\Sigma K N}^{2}}{4 m_{\Sigma}^{2}}\left[\frac{2 m_{\Sigma}\left(m_{\Sigma}+m_{N}\right)+s-m_{\Sigma}^{2}}{s-m_{N}^{2}}\right],
\end{aligned}
$$

and for the $\Xi$ exchange in the diagram $6(d)$

$$
\begin{aligned}
& A_{\Xi}^{+}=\frac{g_{\Sigma K \Xi}^{2}}{4 m_{\Sigma}^{2}}\left(m_{\Xi}+m_{\Sigma}\right)\left(\frac{u-m_{\Sigma}^{2}}{u-m_{\Xi}^{2}}\right), \\
& B_{\Xi}^{+}=\frac{g_{\Sigma K \Xi}^{2}}{4 m_{\Sigma}^{2}}\left[\frac{2 m_{\Sigma}\left(m_{\Sigma}+m_{\Xi}\right)+u-m_{\Sigma}^{2}}{u-m_{\Xi}^{2}}\right], \\
& A_{\Xi}^{-}=-\frac{g_{\Sigma K \Xi}^{2}}{4 m_{\Sigma}^{2}}\left(m_{\Xi}+m_{\Sigma}\right)\left(\frac{u-m_{\Sigma}^{2}}{u-m_{\Xi}^{2}}\right), \\
& B_{\Xi}^{-}=-\frac{g_{\Sigma K \Xi}^{2}}{4 m_{\Sigma}^{2}}\left[\frac{2 m_{\Sigma}\left(m_{\Sigma}+m_{\Xi}\right)+u-m_{\Sigma}^{2}}{u-m_{\Xi}^{2}}\right] .
\end{aligned}
$$

Figure 6(b) gives

$$
\begin{aligned}
& A_{N^{*}}^{+}=\frac{g_{\Sigma K N^{*}}^{2}}{6}\left[\frac{2 \hat{A}+3\left(m_{\Sigma}+m_{N^{*}}\right) t}{m_{N^{*}}^{2}-s}+a_{0}\right], \\
& B_{N^{*}}^{+}=\frac{g_{\Sigma K N^{*}}^{2}}{6}\left[\frac{2 \hat{B}+3 t}{m_{N^{*}}^{2}-s}-b_{0}\right],
\end{aligned}
$$

TABLE III. Resonances of the $K \Sigma$ interaction.

\begin{tabular}{lccc}
\hline \hline & $J^{\pi}$ & $I$ & Mass (MeV) \\
\hline$N$ & $1 / 2^{+}$ & $1 / 2$ & 938 \\
$N(1710)$ & $1 / 2^{+}$ & $1 / 2$ & 1710 \\
$N^{*}(1875)$ & $3 / 2^{-}$ & $1 / 2$ & 1875 \\
$N^{*}(1900)$ & $3 / 2^{+}$ & $1 / 2$ & 1900 \\
$\Delta(1920)$ & $3 / 2^{+}$ & $3 / 2$ & 1920 \\
$\Xi$ & $1 / 2^{+}$ & $1 / 2$ & 1320 \\
$\Xi^{*}(1820)$ & $3 / 2^{-}$ & $1 / 2$ & 1820 \\
\hline \hline
\end{tabular}

$$
\begin{aligned}
& A_{N^{*}}^{-}=\frac{g_{\Sigma K N^{*}}^{2}}{6}\left[\frac{2 \hat{A}+3\left(m_{\Sigma}+m_{N^{*}}\right) t}{m_{N^{*}}^{2}-s}+a_{0}\right], \\
& B_{N^{*}}^{-}=\frac{g_{\Sigma K N^{*}}^{2}}{6}\left[\frac{2 \hat{B}+3 t}{m_{N^{*}}^{2}-s}-b_{0}\right],
\end{aligned}
$$

and for the crossed diagram shown in Fig. 6(e), where a $\Xi^{*}$ exchange is taken into account,

$$
A_{\Xi^{*}}^{+}=\frac{g_{\Sigma K \Xi^{*}}^{2}}{6}\left[\frac{2 \hat{A}^{\prime}+3\left(m_{\Sigma}+m_{\Xi^{*}}\right) t}{m_{\Xi^{*}}^{2}-u}+c_{0}+c_{z}\left(u-m_{\Sigma}^{2}\right)\right],
$$

$B_{\Xi^{*}}^{+}=\frac{g_{\Sigma K \Xi^{*}}^{2}}{6}\left[\frac{2 \hat{B}^{\prime}+3 t}{m_{\Xi^{*}}^{2}-u}-d_{0}-d_{z}\left(u-m_{\Sigma}^{2}\right)\right]$,

$A_{\Xi^{*}}^{-}=-\frac{g_{\Sigma K \Xi^{*}}^{2}}{6}\left[\frac{2 \hat{A}^{\prime}+3\left(m_{\Sigma}+m_{\Xi^{*}}\right) t}{m_{\Xi^{*}}^{2}-u}+c_{0}+c_{z}\left(u-m_{\Sigma}^{2}\right)\right]$,

$B_{\Xi^{*}}^{-}=\frac{g_{\Sigma K \Xi^{*}}^{2}}{6}\left[\frac{2 \hat{B}^{\prime}+3 t}{m_{\Xi^{*}}^{2}-u}-d_{0}-d_{z}\left(u-m_{\Sigma}^{2}\right)\right]$,

where the expressions for $\hat{A}, \hat{B}, \hat{A}^{\prime}, \hat{B}^{\prime}, a_{0}, b_{0}, c_{0}, d_{0}, c_{z}$, and $d_{z}$ are the same ones presented in Sec. III, but replacing the $\Lambda$ hyperon for the $\Sigma$ hyperon.

For the spin-isospin-3/2 $\Delta$ resonance in Fig. 4(c), we have the amplitudes

$$
\begin{aligned}
& A_{\Delta}^{+}=\frac{g_{\Sigma K \Delta}^{2}}{9}\left\{\left[\frac{2 \hat{A^{\prime \prime}}+3\left(m_{\Sigma}+m_{\Delta}\right) t}{m_{\Delta}^{2}-s}\right]+a_{0}^{\prime \prime}\right\}, \\
& B_{\Delta}^{+}=\frac{g_{\Sigma K \Delta}^{2}}{9}\left\{\left[\frac{2 \hat{B^{\prime \prime}}+3 t}{m_{\Delta}^{2}-s}\right]-b_{0}^{\prime \prime}\right\}, \\
& A_{\Delta}^{-}=\frac{g_{\Sigma K \Delta}^{2}}{18}\left\{\left[\frac{2 \hat{A^{\prime \prime}}+3\left(m_{\Sigma}+m_{\Delta}\right) t}{m_{\Delta}^{2}-s}\right]+a_{0}^{\prime \prime}\right\}, \\
& B_{\Delta}^{-}=\frac{g_{\Sigma K \Delta}^{2}}{18}\left\{\left[\frac{2 \hat{B^{\prime \prime}}+3 t}{m_{\Delta}^{2}-s}\right]-b_{0}^{\prime \prime}\right\},
\end{aligned}
$$

where the expressions for $\hat{A}^{\prime \prime}, \hat{B}^{\prime \prime}, a_{0}^{\prime \prime}$ and $b_{0}^{\prime \prime}$ are given in (26), (27), (28) and (29), replacing $\Lambda$ for $\Sigma$ and $N^{*}$ for $\Delta$.

For the $\sigma$ exchange [Fig. 6(f)] the parametrization from Eqs. (36) and (37) will be considered.

Thus, to calculate the observables for each reaction we use (45) and (46), resulting in the amplitudes

$$
\begin{aligned}
& A^{\frac{1}{2}}=A^{+}+2 A^{-}, \\
& B^{\frac{1}{2}}=B^{+}+2 B^{-}, \\
& A^{\frac{3}{2}}=A^{+}-A^{-}, \\
& B^{\frac{3}{2}}=B^{+}-B^{-},
\end{aligned}
$$

and the parameters are shown in Tables II and IV.

To determine the coupling constants $g_{\Sigma K N}$ and $g_{\Sigma K \Xi}$ [Eqs. (40) and (41)] and the ones with resonances we take into account the same arguments presented in Sec. III. 
TABLE IV. Parameters for the $K \Sigma$ interaction.

\begin{tabular}{ll}
\hline \hline$m_{\Sigma}$ & $1190 \mathrm{MeV}$ \\
$g_{\Sigma K N}$ & 6.90 \\
$g_{\Sigma K N(1710)}$ & $6.85 \mathrm{GeV}^{-1}$ \\
$g_{\Sigma K N^{*}(1875)}$ & $0.70 \mathrm{GeV}^{-1}$ \\
$g_{\Sigma K N^{*}(1900)}$ & $1.30 \mathrm{GeV}^{-1}$ \\
$g_{\Sigma K \Delta(1920)}$ & $1.70 \mathrm{GeV}^{-1}$ \\
$g_{\Sigma K \Xi}$ & 13.40 \\
$g_{\Sigma K \Xi^{*}(1820)}$ & $1.80 \mathrm{GeV}^{-1}$ \\
\hline \hline
\end{tabular}

Using the isospin formalism for the elastic and the charge exchange scattering, we can determine the amplitudes for the reactions (that we name $C_{i}$, for simplicity)

$$
\begin{aligned}
\left\langle\Sigma^{+} K^{+}|T| \Sigma^{+} K^{+}\right\rangle & =\left\langle\Sigma^{-} K^{0}|T| \Sigma^{-} K^{0}\right\rangle=T_{\frac{3}{2}} \equiv C_{1} \\
\left\langle\Sigma^{+} K^{0}|T| \Sigma^{+} K^{0}\right\rangle & =\left\langle\Sigma^{-} K^{+}|T| \Sigma^{-} K^{+}\right\rangle \\
& =\frac{1}{3} T_{\frac{3}{2}}+\frac{2}{3} T_{\frac{1}{2}} \equiv C_{2}, \\
\left\langle\Sigma^{0} K^{0}|T| \Sigma^{0} K^{0}\right\rangle & =\left\langle\Sigma^{0} K^{+}|T| \Sigma^{0} K^{+}\right\rangle=\frac{2}{3} T_{\frac{3}{2}}+\frac{1}{3} T_{\frac{1}{2}} \equiv C_{3}, \\
\left\langle\Sigma^{0} K^{0}|T| \Sigma^{-} K^{+}\right\rangle & =\left\langle\Sigma^{+} K^{0}|T| \Sigma^{0} K^{+}\right\rangle \\
& =\left\langle\Sigma^{-} K^{+}|T| \Sigma^{0} K^{0}\right\rangle=\left\langle\Sigma^{0} K^{+}|T| \Sigma^{+} K^{0}\right\rangle \\
& =\frac{\sqrt{2}}{3}\left(T_{\frac{3}{2}}-T_{\frac{1}{2}}\right) \equiv C_{4},
\end{aligned}
$$

and with these amplitudes we can calculate all observables of interest. The total elastic cross sections and the phase shifts as functions of the kaon momentum are shown in Fig. 7.

\section{ANTIKAON-SIGMA INTERACTION}

In this case, we will proceed in the same way as in the last section for the $K \Sigma$ interaction. The diagrams to be considered are shown in Fig. 8.

The Lagrangians are very similar to the ones used to study the $K \Sigma$ interaction, (47) and (48), replacing $N$ and $N^{*}$ for $\Xi$ and $\Xi^{*}$. Then, if these changes are implemented, we may use the same amplitudes given by (49)-(64) and (69)-(72).

In this case we have the following reactions:

$$
\begin{aligned}
\left\langle\bar{K}^{0} \Sigma^{+}|T| \bar{K}^{0} \Sigma^{+}\right\rangle & =\left\langle K^{-} \Sigma^{-}|T| K^{-} \Sigma^{-}\right\rangle=T_{\frac{3}{2}} \equiv D_{1} \\
\left\langle\Sigma^{+} K^{-}|T| \Sigma^{+} K^{-}\right\rangle & =\left\langle\Sigma^{-} \bar{K}^{0}|T| \Sigma^{-} \bar{K}^{0}\right\rangle=\frac{1}{3} T_{\frac{3}{2}}+\frac{2}{3} T_{\frac{1}{2}} \equiv D_{2}, \\
\left\langle\Sigma^{0} \bar{K}^{0}|T| \Sigma^{0} \bar{K}^{0}\right\rangle & =\left\langle\Sigma^{0} K^{-}|T| \Sigma^{0} K^{-}\right\rangle=\frac{2}{3} T_{\frac{3}{2}}+\frac{1}{3} T_{\frac{1}{2}} \equiv D_{3}, \\
\left\langle\Sigma^{0} K^{-}|T| \Sigma^{-} \bar{K}^{0}\right\rangle & =\left\langle\Sigma^{+} K^{-}|T| \Sigma^{0} \bar{K}^{0}\right\rangle \\
& =\left\langle\Sigma^{-} \bar{K}^{0}|T| \Sigma^{0} K^{-}\right\rangle=\left\langle\Sigma^{0} \bar{K}^{0}|T| \Sigma^{+} K^{-}\right\rangle \\
& =\frac{\sqrt{2}}{3}\left(T_{\frac{3}{2}}-T_{\frac{1}{2}}\right) \equiv D_{4} .
\end{aligned}
$$
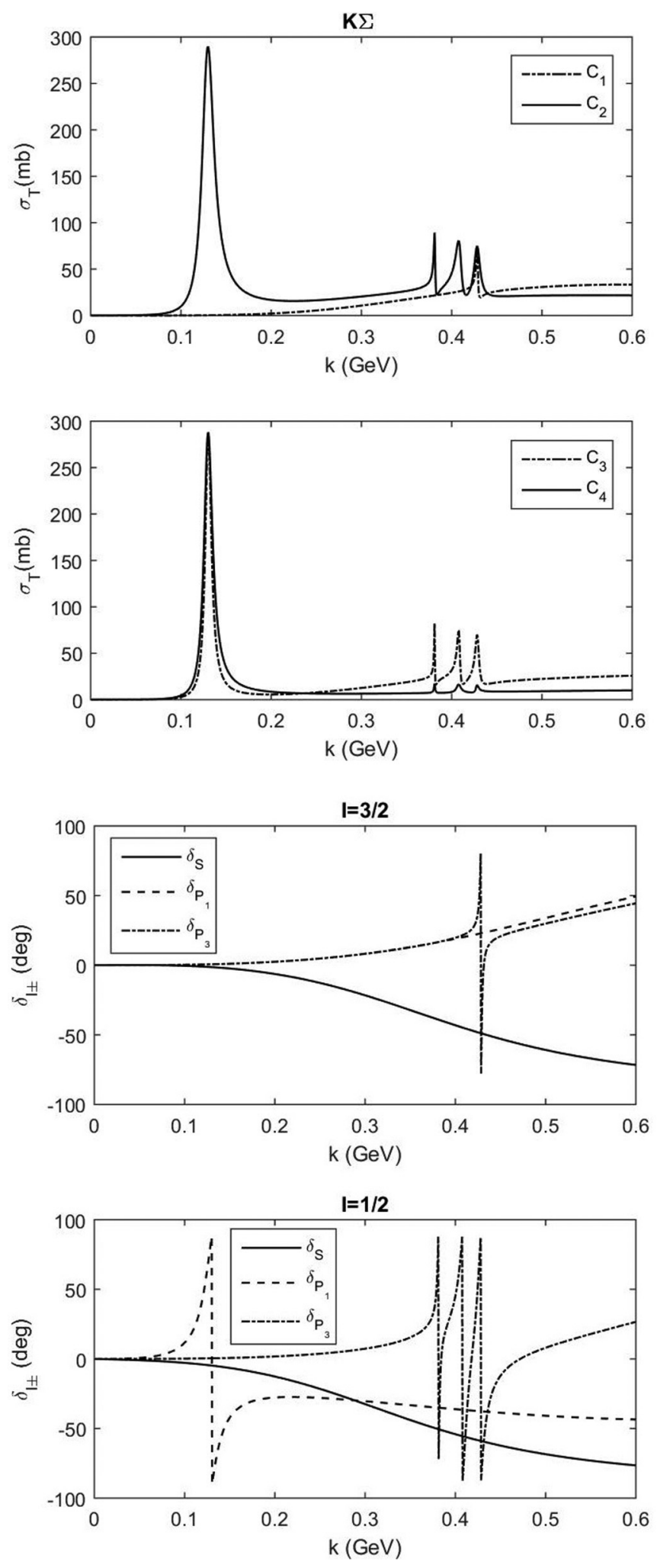

FIG. 7. Total cross section and phase shifts of the $K \Sigma$ scattering.

For diagram $8(\mathrm{c})$ the resonances to be considered are $N(938)$ and $N(1710)$, and for diagram $8(\mathrm{~d})$ the resonance is $N^{*}(1900)$. Using the parameters given in Table IV we obtained the results for the $\bar{K} \Sigma$ scattering shown in Fig. 9. 
(a)

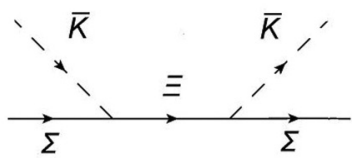

(c)
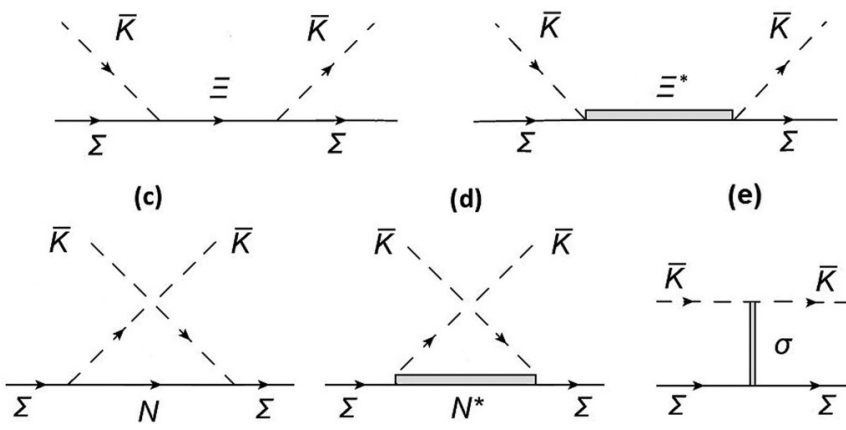

(d)

(e)

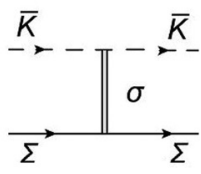

FIG. 8. Diagrams for the $\bar{K} \Sigma$ interaction.

\section{DISCUSSION AND RESULTS}

In this work the low energy $K \Lambda, \bar{K} \Lambda, K \Sigma$, and $\bar{K} \Sigma$ interactions have been studied considering a model based on effective Lagrangians where mesons, baryons, and baryonic resonances have been taken into account. The coupling constants were determined and then the $S$ and $P$ phase shifts, cross sections and polarizations were calculated and shown in the figures of the previous sections. As expected, for many channels the resonances dominated the cross sections, and for this reason we believe in the formulation of the proposed model at low energies $(k<0.4 \mathrm{GeV})$. In [23] a similar behavior were observed, and the predictions of the model, when compared with the HyperCP data, were shown to be very accurate.

For the $\bar{K} \Lambda$ scattering, at the $\Omega$ hyperon mass $\left(m_{\Omega}=\right.$ $1672 \mathrm{MeV}), \delta_{P_{1}}=1.71^{\circ}, \delta_{P_{3}}=5.50^{\circ}, \delta_{D_{3}}=-0.0008^{\circ}$, and $\delta_{D_{5}}=-0.00012^{\circ}$. These strong phases may be used in a possible search of $\mathrm{CP}$ violation in the $\Omega \rightarrow \bar{K} \Lambda$ decay, in addition to the weak $\mathrm{CP}$ violating phases, in the same way as done in [25] (even considering that, for other similar decays, no CP violation has been observed [26,27]).

In the study of high energy hyperon polarization, produced in proton-nucleus and in heavy ion collisions, if we consider the polarizations obtained in the final-state interactions, the processes studied in this work may have some effect in the final polarization of the $\Lambda, \bar{\Lambda}, \Sigma$, and $\bar{\Sigma}$ hyperons produced in these reactions. Especially, in some reactions considerable polarization may be observed, and some signs of this fact may be observed in the $\Sigma$ and in the $\bar{\Sigma}$ polarizations. Probably this effect is smaller than the one obtained when considering the $\pi Y$ interactions $[19,21]$, but as these interactions $(K Y)$ provide polarizations of different signs, it is possible to obtain some differences in the final results. Certainly, a more accurate final result will be obtained.

These reactions are also important in the determination of nucleon-hyperon and hyperon-hyperon potentials, as they are subprocesses of these interactions, and, as discussed before, these interactions have a fundamental importance in the structure of hypernuclei and in hyperon stars. For example, in the reactions $Y Y \rightarrow Y Y$, where $Y$ may be $\Lambda$ or $\Sigma$, the interactions discussed in this paper are part of loop diagrams with $K$ exchange, for example the ones shown in Fig. 10, and a calculation with this procedure may be compared with
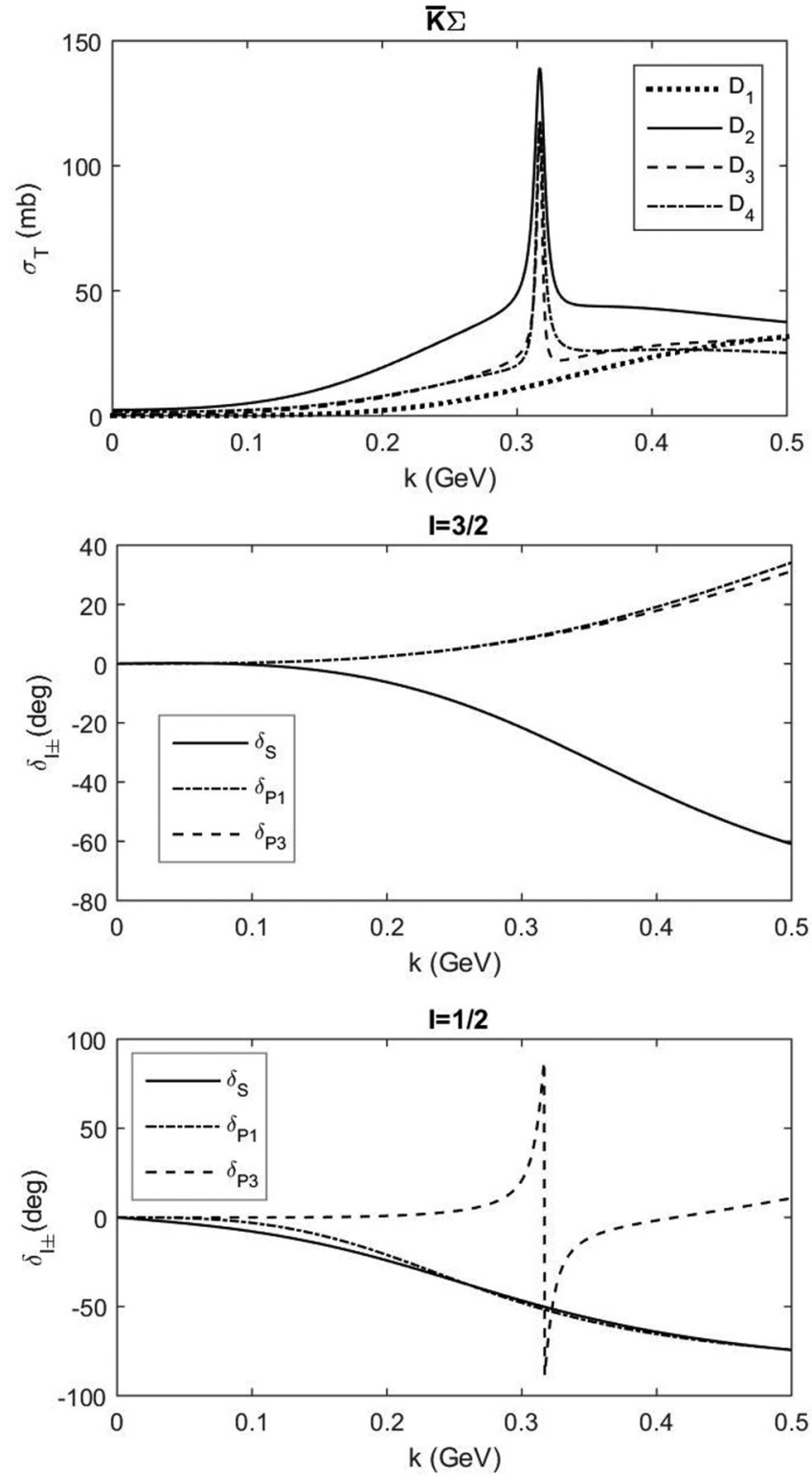

FIG. 9. Total cross section and phase shifts of the $\bar{K} \Sigma$ scattering.

the works of the Nijmegen group. These calculations will be presented in a future work. The $Y Y$ interaction is also related to experiments that seek $S=-2$ hypernuclei.

The study of the $\Xi$ hyperons and other related reactions has been left for future works. (a)

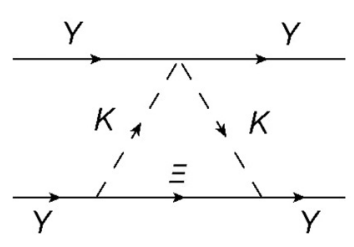

(b)

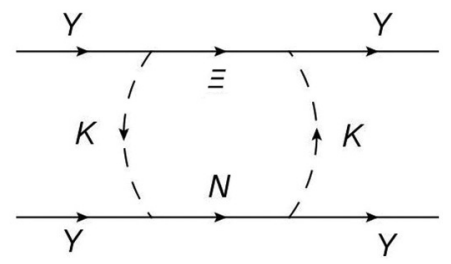

FIG. 10. Examples of kaon exchange in the $Y Y$ interaction. 
We have shown that the study presented in this work is very important for many physical systems of interest, and for this reason it must be continued and improved in future works.

\section{ACKNOWLEDGMENT}

This study was financed in part by the Coordenação de Aperfeiçoamento de Pessoal de Nível Superior - Brasil (CAPES) - Finance Code 001.

\section{APPENDIX}

Considering a process where $p$ and $p^{\prime}$ are the initial and final hyperon four-momenta and $k$ and $k^{\prime}$ are the initial and final meson four-momenta, the Mandelstam variables are given by

$$
\begin{aligned}
& \left.s=(p+k)^{2}=\left(p^{\prime}+k^{\prime}\right)^{2}=m^{2}+m_{K}^{2}+2 E k_{0}-2 \vec{k} \cdot \vec{p} \mathrm{~A} 1\right) \\
& \left.u=\left(p^{\prime}-k\right)^{2}=\left(p-k^{\prime}\right)^{2}=m^{2}+m_{K}^{2}-2 E k_{0}-2 \vec{k}^{\prime} \cdot \vec{p} \mathrm{~A} \text {, }\right) \\
& t=\left(p-p^{\prime}\right)^{2}=\left(k-k^{\prime}\right)^{2}=2|\vec{k}|^{2} x-2|\vec{k}|^{2} .
\end{aligned}
$$

In the center-of-mass frame, the energies will be defined as

$$
\begin{aligned}
& k_{0}=k_{0}^{\prime}=\sqrt{|\vec{k}|^{2}+m_{K}^{2}}, \\
& E=E^{\prime}=\sqrt{|\vec{k}|^{2}+m^{2}},
\end{aligned}
$$

and the total momentum is null,

$$
\vec{p}+\vec{k}=\vec{p}^{\prime}+\vec{k}^{\prime}=0 .
$$

We also define the variable

$$
x=\cos \theta,
$$

where $\theta$ is the scattering angle. Other variables of interest are

$$
\begin{aligned}
v_{r} & =\frac{m_{r}^{2}-m^{2}-k \cdot k^{\prime}}{2 m}, \\
v & =\frac{s-u}{4 m}=\frac{2 E k_{0}+|\vec{k}|^{2}+|\vec{k}|^{2} x}{2 m}, \\
k \cdot k^{\prime} & =m_{K}^{2}+|\vec{k}|^{2}-|\vec{k}|^{2} x=k_{0}^{2}-|\vec{k}|^{2} x,
\end{aligned}
$$

where $m, m_{r}$, and $m_{K}$ are the hyperon mass, the resonance mass and the kaon mass, respectively.

For the energy and the three-momentum of the intermediary particles we also have the relations

$$
\left(E_{B^{*}} \pm m\right)=\frac{\left(m_{B^{*}} \pm m\right)^{2}-m_{K}^{2}}{2 m_{B^{*}}}
$$

$$
\left(q_{B^{*}}\right)^{2}=\left|\vec{q}_{B^{*}}\right|^{2}=E_{B^{*}}^{2}-m^{2}=\left(E_{B^{*}}+m\right)\left(E_{B^{*}}-m\right),
$$

where $E_{B^{*}}$ and $\vec{q}_{B^{*}}$ are the energy and the momentum of intermediary baryon $B^{*}$ in the center-of-mass frame, respectively.
[1] R. S. Hayano et al., Phys. Lett. B 231, 355 (1989).

[2] T. Nagae et al., Phys. Rev. Lett. 80, 1605 (1998).

[3] S. Bart et al., Phys. Rev. Lett. 83, 5238 (1999).

[4] J. Schaffner, C. Greiner, and H. Stöcker, Phys. Rev. C 46, 322 (1992).

[5] N. K. Glendenning, Astrophys. J. 293, 470 (1985).

[6] J. Schaffner-Bielich, Nucl. Phys. A 804, 309 (2008).

[7] M. Baldo, G. F. Burgio, and H. J. Schulze, Phys. Rev. C 61, 055801 (2000).

[8] I. Vidana, A. Polls, A. Ramos, L. Engvik, and M. Hjorth-Jensen, Phys. Rev. C 62, 035801 (2000).

[9] B. I. Abelev et al. (STAR Collaboration), Phys. Rev. C 76, 024915 (2007).

[10] G. Bunce et al., Phys. Rev. Lett. 36, 1113 (1976).

[11] K. Heller et al., Phys. Lett. B 68, 480 (1977); Phys. Rev. Lett. 41, 607 (1978); S. Erhan et al., Phys. Lett. B 82, 301 (1979).

[12] B. Lundberg et al., Phys. Rev. D 40, 3557 (1989).

[13] C. Wilkinson et al., Phys. Rev. Lett. 46, 803 (1981).

[14] L. Deck et al., Phys. Rev. D 28, 1 (1983).

[15] R. Ramerika et al., Phys. Rev. D 33, 3172 (1986).

[16] M. I. Adamovich et al., Z. Phys. A 350, 379 (1995).

[17] P. M. Ho et al., Phys. Rev. Lett. 65, 1713 (1990); Phys. Rev. D 44, 3402 (1991).

[18] A. Morelos et al., Phys. Rev. Lett. 71, 2172 (1993); Phys. Rev. D 52, 3777 (1995).

[19] C. C. Barros, Jr. and Y. Hama, Int. J. Mod. Phys. E 17, 371 (2008).

[20] C. C. Barros, Jr. and Y. Hama, Phys. Lett. B 699, 74 (2011).
[21] C. C. Barros, Jr., J. Phys.: Conf. Ser. 509, 012056 (2014).

[22] L. Adamczyk et al. (STAR Collaboration), Nature (London) 548, 62 (2017).

[23] C. C. Barros, Jr. and Y. Hama, Phys. Rev. C 63, 065203 (2001).

[24] C. C. Barros, Jr. and M. R. Robilotta, Eur. Phys. J. C 45, 445 (2006)

[25] C. C. Barros, Jr., Phys. Rev. D 68, 034006 (2003).

[26] A. Chakravorty et al., Phys. Rev. Lett. 91, 031601 (2003).

[27] M. Huang et al., Phys. Rev. Lett. 93, 011802 (2004).

[28] H. T. Coelho, T. K. Das, and M. R. Robilotta, Phys. Rev. C 28 , 1812 (1983).

[29] H. Pilkuhn, The Interaction of Hadrons (North-Holland, Amsterdam, 1967).

[30] E. T. Osypowski, Nucl. Phys. B 21, 615 (1970).

[31] M. G. Olsson and E. T. Osypowski, Nucl. Phys. B 101, 136 (1975).

[32] C. Patrignani et al., Chin. Phys. C 40, 100001 (2016).

[33] J. Gasser, M. E. Sainio, and A. Švarc, Nucl. Phys. B 307, 779 (1988); T. Becher and H. Leutwyler, Eur. Phys. J. C 9, 643 (1999); J. High Energy Phys. 06 (2001) 017.

[34] J. Gasser, H. Leutwyler, and M. E. Sainio, Phys. Lett. B 253, 252 (1991); 253, 260 (1991).

[35] A. I. L'vov, S. Scherer, B. Pasquini, C. Unkmeir, and D. Drechsel, Phys. Rev. C 64, 015203 (2001).

[36] M. R. Robilotta, Phys. Rev. C 63, 044004 (2001).

[37] V. Stoks, T. A. Rijken, Nucl. Phys. A 613, 311 (1997).

[38] J. D. Swart, Rev. Mod. Phys. 35, 916 (1963).

[39] T. Ericson and W. Weise, Pions and Nuclei (Oxford University Press, Oxford, 1988).

[40] H. Pilkuhn et al., Nucl. Phys. B 65, 460 (1973). 